\title{
Critérios diagnósticos e tratamento dos distúrbios respiratórios do sono: RERA
}

\author{
Diagnostic criteria and treatment for sleep-disordered breathing: RERA
}

Luciana de Oliveira Palombini

\begin{abstract}
Resumo
Na polissonografia, RERA é definido como um parâmetro respiratório que indica um despertar associado a um evento respiratório e um aumento do esforço respiratório. Inicialmente, RERA foi descrito com o uso da manometria esofágica utilizada para avaliação do esforço respiratório. Esse maior esforço respiratório ocorre como resposta a um aumento da resistência da via aérea superior, aspecto presente na fisiopatologia dos distúrbios respiratórios do sono, entre esses, SAOS e SRVAS. Posteriormente, o uso de cânula de pressão nasal foi relatado como uma maneira confiável e mais sensível que o termistor para a identificação de eventos de redução do fluxo aéreo e também como um substituto da manometria esofágica para a identificação de períodos de aumento da resistência na via aérea superior. Consequentemente, a American Academy of Sleep Medicine recomenda o uso de um dos métodos para a identificação de RERA, que é definido por um padrão de achatamento da curva inspiratória, característico da limitação ao fluxo aéreo. Embora RERA seja identificado e avaliado na pratica médica, sua padronização ainda é necessária. Portanto, recomenda-se que os laudos de polissonografia indiquem quais eventos respiratórios anormais foram considerados na avaliação do grau de gravidade do distúrbio respiratório.
\end{abstract}

Descritores: Síndromes da apneia do sono; Resistência das vias respiratórias; Manometria.

\begin{abstract}
In polysomnography, RERA is defined as a respiratory parameter that indicates an arousal associated with a respiratory event and an increase in respiratory effort. Initially, RERA was described by means of esophageal manometry for the evaluation of respiratory effort. This greater respiratory effort occurs as a response to an increase in upper airway resistance, which is a factor present in the pathophysiology of sleep-disordered breathing, such as obstructive sleep apnea syndrome and upper airway resistance syndrome. Later, the use of a nasal pressure cannula was reported to be a reliable means of identifying airflow limitation and one that is more sensitive than is a thermistor. In addition, the nasal pressure cannula method has been used as a surrogate for esophageal manometry in the identification of periods in which upper airway resistance increases. Consequently, the American Academy of Sleep Medicine recommend the use of either method for the identification of RERA, which is defined by the flattening of the inspiratory curve, characteristic of airflow limitation. Although RERA has been identified and evaluated in current medical practice, it has yet to be standardized. Therefore, it is recommended that polysomnographic reports indicate which abnormal respiratory events were taken into consideration in the evaluation of the severity of sleep-disordered breathing.
\end{abstract}

Keywords: Sleep apnea syndromes; Airway resistance; Manometry.

\section{Critérios diagnósticos: RERA}

Inicialmente descrito pela American Academy of Sleep Medicine, RERA foi definido como um evento respiratório caracterizado por um aumento do esforço respiratório levando ao despertar do sono, mas que não fecha com os critérios para apneia e hipopneia.(1) 0 evento deveria preencher os seguintes critérios: padrão de pressão negativa progressivamente mais negativa, terminado por uma súbita mudança de pressão para um nível menos negativo, e um despertar; o evento deve ter uma duração mínima de $10 \mathrm{~s}$ (Figura 1). A partir desse estudo, o evento de RERA foi incluído na contagem de eventos respiratórios anormais, em conjunto com apneias e hipopneias para a identificação de SAOS. A polissonografia noturna deve demonstrar cinco ou mais eventos obstrutivos por hora de sono, sendo que esses eventos podem ser apneias, hipopneias ou RERAs para a definição do diagnóstico de SAOS.

Inicialmente, RERA foi descrito apenas com o uso da pressão esofágica para a identificação de maior esforço respiratório associado à maior resistência da via aérea superior. Posteriormente, foi incluído o achatamento da curva de fluxo 


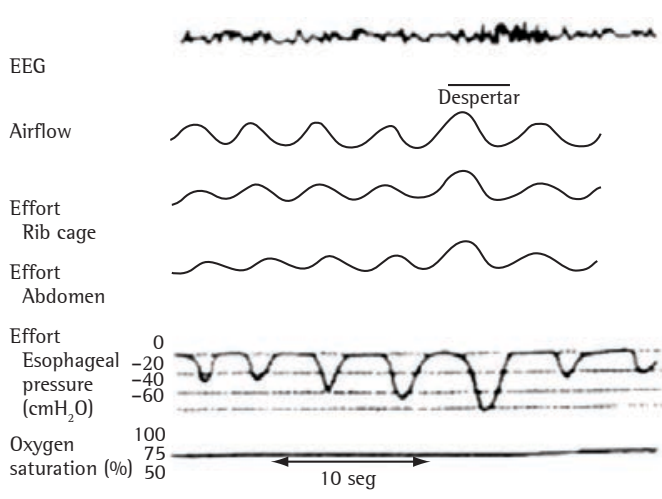

Figura 1 - Pressão esofágica crescente, aumento progressivo da pressão esofágica sem alteração significativa do fluxo aéreo, levando a despertar no EEG.

aéreo demonstrado com uso de cânula de pressão nasal.(2)

\section{Esforço respiratório durante o sono}

0 principal aspecto no reconhecimento de SRVAS na polissonografia é o aumento do esforço respiratório durante o sono na ausência de um número significativo de apneias e hipopneias que estabeleça um $1 \mathrm{AH}>5$ eventos/h de sono. 0 esforço respiratório pode ser avaliado de diversas maneiras. A descrição inicial de SRVAS foi feita a partir da avaliação do esforço respiratório durante o sono com o uso de manometria esofágica. ${ }^{(3)}$ Posteriormente, o uso de um tubo nasogástrico pediátrico conectado a um transdutor de pressão $0^{(4)}$ substituiu o balão para medida da pressão esofágica.

A determinação de valores anormais da pressão esofágica depende de cada pessoa, pois um valor de $-7 \mathrm{CmH}_{2} \mathrm{O}$ pode ser associado à fragmentação do sono e a queixas de sonolência em uma pessoa de porte médio, mas pode não levar a consequências importantes em outro individuo de estrutura maior. Um grupo de autores ${ }^{(5)}$ descreveu padrões de pressão negativos considerados indicativos de esforço respiratório anormal: pressão esofágica crescente, esforço aumentado contínuo e reversão da pressão esofágica.

A desvantagem do uso da pressão esofágica é a necessidade da inserção de um cateter nasal na narina dos pacientes até o nível do esôfago. Apesar da demonstração de boa tolerância e de baixa frequência de complicações em adultos e crianças, a medida de pressão esofágica não é amplamente utilizada devido ao desconforto provocado aos pacientes. Entretanto, a medida da pressão esofágica é ainda o padrão ouro na avaliação do esforço respiratório.

Outros métodos polissonográficos que têm sido relatados como indicadores de aumento da resistência de vias aéreas superiores citados na literatura incluem a pletismografia de impedância ${ }^{(6)}$ a técnica da oscilação forçada ${ }^{(7)} \mathrm{e}$ pressão crítica. ${ }^{(8)}$

Em 1999, RERA foi oficialmente descrito e definido como o registro de esforço respiratório aumentado associado a um despertar. Nesse caso, portanto, a presença de um despertar como descrito pela American Academy of Sleep Medicine (1992) é necessária. Posteriormente, foi incluído o padrão de achatamento da curva de fluxo aéreo identificada na cânula nasal com transdutor de pressão. Esse achatamento de curva de fluxo, no entanto, não preenche os critérios para eventos de hipopneia, mas, do mesmo modo, deve ser seguido de um despertar breve no EEG.

\section{Limitação ao fluxo aéreo}

0 padrão respiratório de achatamento da curva de fluxo aéreo presente em eventos de RERA indica a presença de limitação ao fluxo aéreo. Essa limitação acontece quando o aumento da pressão negativa esofágica não é acompanhado por um aumento proporcional no fluxo aéreo. A limitação ao fluxo aéreo depende da interação entre a pressão pleural negativa, a qual tende a colapsar a via aérea superior, e o aumento da atividade de músculo de via aérea superior, que tende a dilatar a mesma. Essa falta de linearidade na relação pressão-fluxo durante a inspiração é comumente causada pelo estreitamento de uma via aérea superior hipotônica em resposta à pressão negativa intratorácica desenvolvida durante a inspiração. A presença de limitação ao fluxo aéreo tem sido usada como um indicador de aumento da resistência da via aérea superior e tem sido utilizada como parâmetro para a avaliação de SRVAS. ${ }^{(2)}$ É recomendado, ao utilizar-se a cânula de pressão nasal como indicador de limitação ao fluxo aéreo, acrescentar-se um termistor na região da boca para a avaliação da respiração oral associada à obstrução nasal. 


\section{Periodos prolongados de limitação ao fluxo aéreo}

Períodos curtos de limitação ao fluxo aéreo são aceitos hoje como eventos respiratórios anormais. Além de RERA (períodos de limitação ao fluxo aéreo de $10 \mathrm{~s}$ ), períodos prolongados de limitação ao fluxo aéreo também têm sido demonstrados em alguns trabalhos, ${ }^{(9)}$ mas 0 significado desses eventos ainda não está bem esclarecido (Figura 2).

\section{Tratamento}

0 tratamento dos distúrbios respiratórios do sono envolve o tratamento de SAOS, SRVAS e ronco. 0 tratamento de SAOS e de ronco deve seguirasindicações clínicas citadasanteriormente. No caso de RERA, esses podem estar presentes em distúrbios respiratórios do sono, entre eles SAOS e SRVAS, ou seja, o tratamento dessa anormalidade respiratória está envolvido no tratamento dos distúrbios respiratórios do sono. Já o tratamento de SRVAS é mais controverso.
$\mathrm{Na}$ descrição inicial, ${ }^{(10)}$ os pacientes apresentaram reversão das queixas após o uso de CPAP por 30 dias. Apesar de ser hoje considerado uma opção terapêutica, a adesão para o uso de CPAP é baixa. ${ }^{(11)}$ A cirurgia também pode ser indicada em algumas situações, dependendo das anormalidades anatômicas presentes na via aérea superior. Técnicas descritas para esses pacientes incluem septoplastia, radiofrequência e distração osteogênica em pacientes com SRVAS. ${ }^{(12)} 0$ uso de aparelho intraoral também foi avaliado como forma de tratamento de SRVAS, ${ }^{(13)}$ mas há poucos estudos a esse respeito.

\section{Considerações finais}

0 reconhecimento dos diferentes tipos de eventos respiratórios anormais na polissonografia é essencial na identificação dos distúrbios respiratórios do sono. Além de apneias e hipopneias, RERA deve ser identificado, pois indica uma diminuição do espaço da via aérea superior.



Figura 2 - Período prolongado de limitação ao fluxo aéreo. 
Embora RERA descreva uma anormalidade polissonográfica respiratória indicativa de estreitamento de via aérea superior, esse não alcança os critérios de hipopneia. A diferenciação entre esses dois parâmetros é difícil, pois, atualmente, essa avaliação é baseada no aspecto qualitativo do sinal do fluxo aéreo. Provavelmente novas tecnologias irão possibilitar o reconhecimento dessa anormalidade de forma quantitativa e, com isso, possibilitar uma padronização em relação à ocorrência de RERA em pacientes com distúrbios respiratórios do sono. Hoje, a identificação da limitação ao fluxo aéreo esta limitada à definição de RERA, mas sabe-se, a partir da prática clínica, que períodos de limitação ao fluxo aéreo ocorrem em diferentes situações e apresentações. Portanto, são necessários novos estudos para 0 estabelecimento de normas para a classificação das anormalidades respiratórias presentes em pacientes com distúrbios respiratórios do sono e que fogem aos critérios de apneias e hipopneias.

\section{Referências}

1. Sleep-related breathing disorders in adults: recommendations for syndrome definition and measurement techniques in clinical research. The Report of an American Academy of Sleep Medicine Task Force. Sleep. 1999;22(5):667-89.

2. Ayappa 1, Norman RG, Krieger AC, Rosen A, O'malley RL, Rapoport DM. Non-Invasive detection of respiratory effort-related arousals (REras) by a nasal cannula/ pressure transducer system. Sleep. 2000;23(6):763-71.
3. Flemale A, Gillard C, Dierckx JP. Comparison of central venous, oesophageal and mouth occlusion pressure with water-filled catheters for estimating pleural pressure changes in healthy adults. Eur Respir J. 1988;1(1):51-7.

4. Chervin RD, Burns JW, Ruzicka DL. Electroencephalographic changes during respiratory cycles predict sleepiness in sleep apnea. Am J Respir Crit Care Med. 2005;171(6):652-8.

5. Guilleminault C, Poyares D, Palombini L, Koester U, Pelin Z, Black J. Variability of respiratory effort in relation to sleep stages in normal controls and upper airway resistance syndrome patients. Sleep Med. 2001;2(5):397-405.

6. Loube Dl, Andrada T, Howard RS. Accuracy of respiratory inductive plethysmography for the diagnosis of upper airway resistance syndrome. Chest. 1999;115(5):1333-7.

7. Rühle KH, Schlenker E, Randerath W. Upper airway resistance syndrome. Respiration. 1997;64 Suppl 1:29-34.

8. Gold AR, Schwartz AR. The pharyngeal critical pressure. The whys and hows of using nasal continuous positive airway pressure diagnostically. Chest. 1996;110(4):107788.

9. Calero G, Farre R, Ballester E, Hernandez L, Daniel $\mathrm{N}$, Montserrat Canal JM. Physiological consequences of prolonged periods of flow limitation in patients with sleep apnea hypopnea syndrome. Respir Med. 2006;100(5):813-7.

10. Guilleminault C, Stoohs R, Clerk A, Cetel M, Maistros $P$. A cause of excessive daytime sleepiness. The upper airway resistance syndrome. Chest. 1993;104(3):781-7.

11. Rauscher H, Formanek D, Zwick H. Nasal continuous positive airway pressure for nonapneic snoring? Chest. 1995;107(1):58-61.

12. Guilleminault C, Li KK. Maxillomandibular expansion for the treatment of sleep-disordered breathing: preliminary result. Laryngoscope. 2004;114(5):893-6.

13. Yoshida K. Oral device therapy for the upper airway resistance syndrome patient. J Prosthet Dent. 2002;87(4):427-30.

\section{Sobre a autora}

\section{Luciana de Oliveira Palombini}

Médica Pneumologista e Coordenadora do Instituto do Sono de São Paulo, São Paulo (SP) Brasil.

E-mail para contato: Ipalombini@hotmail.com (L. Palombini) 